\title{
El tratamiento intralesional de Leishmaniasis cutánea es seguro frente al riesgo de complicaciones mucosas?
}

\author{
Is the intralesional treatment of cutaneous Leishmaniasis safe against the risk of mucosal complications?
}

\author{
Jhonny Wilson Limachi-Choque ${ }^{1 a}$, Ernesto Rojas-Cabrera ${ }^{2 a}$, Aleida Verduguez-Orellana ${ }^{2 b}$, Daniel Eid-Rodríguez ${ }^{2 c}$
}

\author{
Resumen
}

Objetivo: evaluar la seguridad a largo plazo frente al riesgo de complicaciones mucosas del uso intralesional de antimoniales pentavalentes en pacientes con Leishmaniasis cutánea comparado con el uso sistémico de los mismos. Métodos: estudio observacional, cuantitativo de tipo longitudinal retrospectivo. Se analizó un total de 66 registros clínicos de pacientes, con diagnóstico de Leishmaniasis cutánea del parque Isiboro Secure durante el periodo 2012 a 2016. Se evaluó un total de 46 tratamientos sistémicos y 20 intralesionales. Resultados: la evaluación clínica realizada entre 4 y 7 años posteriores a la cicatrización de las lesiones cutáneas de Leishmaniasis mostró la ausencia de desarrollo de lesiones mucosas. Así mismo no se reportó fallas terapéuticas, recidivas ni efectos adversos a corto plazo. Conclusiones: el tratamiento intralesional fue seguro y eficaz a largo plazo y es una opción confiable para el tratamiento de Leishmaniasis cutánea evitando las complicaciones futuras de la enfermedad.

Palabras claves: Leishmaniasis mucosa, tratamiento intralesional, tratamiento sistémico

\section{Abstract}

Objective: to assess the long-term safety against the risk of mucosal complications of intralesional pentavalent antimonials (PA) in patients with cutaneous leishmaniasis compared to the systemic use of PA. Methods: retrospective longitudinal quantitative observational study. A total of 66 clinical records of patients diagnosed with cutaneous leishmaniasis in Isiboro Secure Park were analyzed between 2012 and 2016. A total of 46 systemic and 20 intralesional treatments were evaluated. Results: clinical evaluation 4-7 years after healing of leishmaniasis skin lesions showed no development of mucosal lesions. Likewise, no therapeutic failures, relapses or short-term adverse effects were reported. Conclusions: intralesional treatment was safe and effective in the long term and is a reliable option for the treatment of cutaneous leishmaniasis avoiding the future complications of the disease.

Keywords: mucosal leishmaniasis, intralesional treatment, systemic treatment, treatment safety

$\mathrm{L}^{2}$ a Leishmaniasis es una enfermedad parasitaria, no contagiosa, causada por diferentes especies de protozoos del género Leishmania ${ }^{1}$. La OMS, estima una incidencia de Leishmaniasis aproximada de 2 millones de casos nuevos por $\mathrm{año}^{2}$, con defunciones por esta causa que llegan a las 67000 por año en todo el mundo ${ }^{3}$. En Sud América, la enfermedad está presente en casi todos los países excepto Chile y Uruguay $^{3}$. La Leishmaniasis presenta tres formas clínicas: la visceral, la cutánea y la mucocutánea. La forma cutánea es la más frecuente y consiste en ulceras crónicas que dejan cicatrices de por vida, y la forma mucocutánea, más agresiva y produce la destrucción parcial o completa de las membranas mucosas de nariz, boca y laringe ${ }^{3}$. En Bolivia, la especie más frecuente y responsable del $85 \%$ de los casos de Leishmaniasis cutánea es L. braziliensis ${ }^{4}$. Esta especie también es responsable de la Leishmaniasis mucosa, la que generalmente ocurre posterior a un episodio de leismaniasis cutánea debido a una diseminación sistémica del parásito ${ }^{5}$.

Los antimoniales pentavalentes son los medicamentos

${ }^{1}$ Medico, Mg. en Salud Pública,

${ }^{2}$ Centro Universitario de Medicina Tropical, UMSS

${ }^{a}$ Médico Especialista en Medicina Tropical.

bioquimica, MSc. en Epidemiologia Clínica.

'PhD. En Salud Pública

${ }^{*}$ Correspondencia a: Jhonny Wilson Limachi-Choque

Correo electrónico: Jhon.traumato@gmail.com

Recibido el 24 de diciembre de 2019. Aceptado el 23 de marzo de 2020 de primera línea para el tratamiento de la Leishmaniasis cutánea y se administran sistémicamente (vía endovenosa o intramuscular) a una dosis de $20 \mathrm{mg} / \mathrm{kg} /$ día por 20 días. Según un meta análisis realizado el 2008, este tratamiento tiene una eficacia de curación clínica del 76,5\% ${ }^{7}$. Sin embargo, esta terapia tiene como desventaja el empleo de múltiples inyecciones y grandes volúmenes de medicamento, provocando efectos adversos como mialgias, artralgias, síntomas gastrointestinales y toxicidad importante a nivel cardiaco, hepático, pancreático y renal. Las reacciones adversas y la toxicidad asociada a este tratamiento son responsables de abandono del tratamiento de parte del paciente, así como a interrupciones parciales o totales del tratamiento de parte del personal médico, situaciones que dan lugar a problemas de falla terapéutica que son mucho más complicados de manejar ${ }^{8,9}$.

La aplicación intralesional de antimoniales pentavalentes es una técnica ampliamente utilizada para tratar la Leishmaniasis en el mundo con una curación clínica del $70 \%{ }^{7}$, por lo cual es recomendada actualmente por la OPS/ $\mathrm{OMS}^{6}$; es por ello que Brasil, que tiene la misma cepa responsable de Leishmaniasis mucosa, ha incluido un manual de manejo para el uso de antimoniales intralesionales para la enfermedad ${ }^{10}$. Adicionalmente, en Bolivia nuestro grupo de trabajo, ha desarrollado experiencias propias en este tipo de taratamiento ${ }^{4}$, sin embargo, todavía este tratamiento no ha sido suficientemente aceptado, ni implementado en Bolivia 
por parte de las autoridades del ministerio de salud, por una excesiva desconfianza respecto al éxito de este tratamiento, argumentando las escasas evidencias para respaldar la seguridad del tratamiento ${ }^{11}$. Con el interés de contribuir con más evidencias sobre este particular, el objetivo de este estudio fue evaluar la seguridad del tratamiento intralesional con antimoniales pentavalentes de Leishmaniasis cutánea, frente al riesgo de complicaciones mucosas a largo plazo.

\section{Material y métodos}

El presente estudio observacional, es cuantitativo de tipo longitudinal retrospectivo. Se consideraron criterios de exclusión en el estudio el haber realizado otros tipos de tratamiento diferentes al tratamiento intralesional o sistémico y la imposibilidad de realizar seguimiento del postratamiento. Por otra parte, se consideró como criterios de inclusión el haber recibido tratamiento basado en antimoniales pentavalentes (intralesionales o sistémicos) dentro del periodo de seguimiento y su aceptación de participar voluntariamente en el estudio.

Sesenta y seis registros clínicos fueron seleccionados para el estudio de pacientes con diagnóstico confirmado por laboratorio de Leishmaniasis cutánea que recibieron el tratamiento intralesional o el tratamiento sistémico con anitmoniales pentavalentes durante el periodo 2012 a 2016 y que correspondían a alguna comunidad del parque Isiboro Sécure en el Municipio de Villa Tunari. De los 66 pacientes, 46 de ellos recibieron el tratamiento sistémico y 20 recibieron tratamiento intralesional. Este último consistió en la aplicación local de 3 y 6 dosis de antimoniales pentavalentes en el borde de la lesión en una frecuencia de día por medio. La cantidad de medicamento aplicado fue calculado multiplicando el ancho por el largo de la lesión y por un factor constante de 0,008, de acuerdo con la guía operativa de Bolivia ${ }^{4}$ El tratamiento intralesional se realizó en base a dos esquemas: 9 pacientes recibieron tres aplicaciones en un primer ensayo clínico en las gestiones 2012-2013 teniendo un seguimiento de 7 años, y los otros 11 recibieron seis aplicaciones como parte de un segundo ensayo clínico realizado entre las gestiones 20142015 con un seguimiento de 4 años $^{4,5}$.

\section{Recolección de datos}

La información clínica de los participantes del estudio se recolecto de los registros disponibles en los centros de salud distribuidos en el territorio del parque Isiboro Sécure, conjuntamente con una nueva evaluación clínica "in situ” de los pacientes previamente notificados para este propósito.

Evaluación clínica posterior a la cicatrización de la lesión los participantes fueron evaluados por medio de una exploración física completa en busca de complicaciones que pudieran presentarse posterior a la culminación del tratamiento. Específicamente se evaluó: 1) la presencia de actividad parasitaria (caracterizada por reaparición de la úlcera o inflamación de los bordes de la cicatriz); 2) la aparición de un nuevo episodio en áreas de la piel distintas a donde se encontraba la lesión cicatrizada; y 3) la presencia de ulceras en mucosas de nariz, boca, cambios en tonalidad de la voz como señal de complicación mucosa.

\section{Consideraciones éticas}

El estudio fue aprobado por el Comité de Ética Boliviano de la Facultad de Medicina de la Universidad Mayor de San Simón. Todos los participantes del estudio dieron su consentimiento voluntario por escrito aceptando participar en el estudio.

Tabla 1. Datos demográficos y clínicos de los pacientes con leishmaniasis cutánea que recibieron tratamiento sistémico o intralesional con antimoniales pentavalentes. Datos presentados como frecuencias (\%).

\begin{tabular}{|c|c|c|c|c|}
\hline \multicolumn{2}{|c|}{ Variables } & \multirow{2}{*}{$\begin{array}{c}\text { Tx. sistémico } \\
\mathrm{n}=46 \\
28(4-67)\end{array}$} & $\underset{n=11}{\mathrm{~A}}$ & $\underset{n=9}{B}$ \\
\hline Edad (años) ${ }^{\mathrm{a}}$ & & & $29(14-52)$ & $22(6-36)$ \\
\hline \multirow{2}{*}{ Genero } & M & $22(47,8)$ & $2(18,2)$ & $5(55,6)$ \\
\hline & F & $24(52,2)$ & $9(81,8)$ & $4(44,4)$ \\
\hline \multirow{4}{*}{ Ocupación } & Agricultura & $19(41,3)$ & $2(18,2)$ & $5(55,6)$ \\
\hline & Estudiante & $16(34,8)$ & $4(36,4)$ & $4(44,4)$ \\
\hline & Labores de casa & $9(18,6)$ & $4(36,4)$ & $\neg$ \\
\hline & Otros & $2(4,3)$ & $1(9,1)$ & $\neg$ \\
\hline \multirow{3}{*}{$\begin{array}{l}\text { Región anatómica } \\
\text { comprometida }\end{array}$} & Cabeza/cuello & $5(10,8)$ & $\neg$ & $\neg$ \\
\hline & Extrem, Sup, & $9(19,6)$ & $1(9,1)$ & $1(11,1)$ \\
\hline & Extrem, Inf, & $32(4,3)$ & $10(90,9)$ & $8(88,9)$ \\
\hline \multirow{3}{*}{ Número de lesiones } & Una & $36(78,3)$ & $9(81,2)$ & $7(77,8)$ \\
\hline & Dos & $7(15,3)$ & $2(18,2)$ & $2(22,2)$ \\
\hline & $>$ a dos & $3(6,5)$ & $\neg$ & $\neg$ \\
\hline \multicolumn{2}{|c|}{ Demora de inicio de Tratamiento en semanas ${ }^{a}$} & $10(2-39)$ & $5(4-13)$ & $11(3-34)$ \\
\hline \multirow{2}{*}{ Enfermedad concomitante } & $\mathrm{Si}$ & $1(2,2)$ (anemia) & $1(9,1)$ (Chagas) & $\neg$ \\
\hline & No & $45(97,8)$ & $10(90,9)$ & $9(100)$ \\
\hline
\end{tabular}

aPromedio (Rango); Tx Sistémico = Tratamiento convencional por 20 días de aplicación intramuscular dosis/kg/peso; Tx. intralesional (A) = Seis aplicaciones de medicamento intralesional; Tx intralesional $(B)=$ Tres aplicaciones de medicamento intralesional 
Figura 1. Área de la lesión de pacientes con leishmaniasis cutánea antes y después de la intervención terapéutica. Tx. Sistémico = Tratamiento convencional por 20 días de aplicación intramuscular dosis $/ \mathrm{kg} /$ peso $(\mathrm{n}=46)$; Tx. Intralesional $(A)=$ Seis aplicaciones de medicamento Intralesional $(n=9)$; Tx Intralesional $(B)=$ Tres aplicaciones de medicamento Intralesional $(n=11)$. Datos expresados como Mediana (RIC).

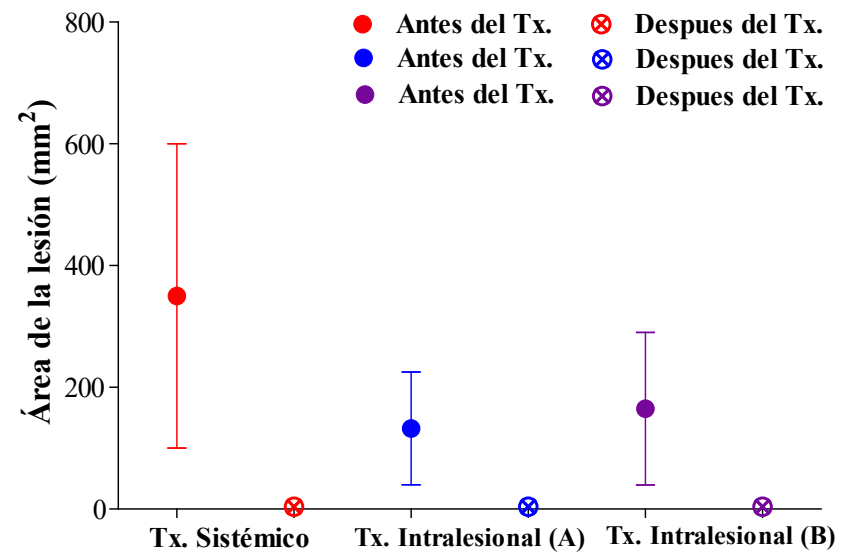

\section{Resultados}

Fueron contactados sesenta y seis pacientes con Leishmaniasis cutánea que recibieron tratamiento sistémico o intralesional entre 2012 y 2016. De este total, el mayor porcentaje eran mujeres y el rango de edades estaba comprendida entre 4 a 67 años de edad, con una media de 26 años (Tabla1).

Todos ellos, alcanzaron la cicatrización de sus ulceras cutáneas dentro de los 6 meses de seguimiento posterior a la conclusión del tratamiento, independientemente del tipo de tratamiento recibido (Figura 1). Se debe señalar que el área de la ulcera era menor a $900 \mathrm{~mm}^{2}(30 \times 30 \mathrm{~mm})$ en todos los casos incluidos en el estudio (tratamiento sistémico o intralesional). El tiempo en el que las lesiones alcanzaron la cicatrización, de acuerdo con los reportes revisados estaban en el rango de los 6 meses posteriores a la finalización del tratamiento.
Figura 2. Seguimiento clínico en busca de lesiones mucosas por Leishmania, posterior a la curación clínica (cicatriz) de leishmaniasis cutánea. Tx sistémico = Tratamiento convencional por 20 días de aplicación intramuscular dosis / kg/peso $(n=46)$; Tx. intralesional $(A)=$ Seis aplicaciones de medicamento intralesional $(n=9)$; Tx. intralesional $(B)=$ Tres aplicaciones de medicamento intralesional $(n=11)$.

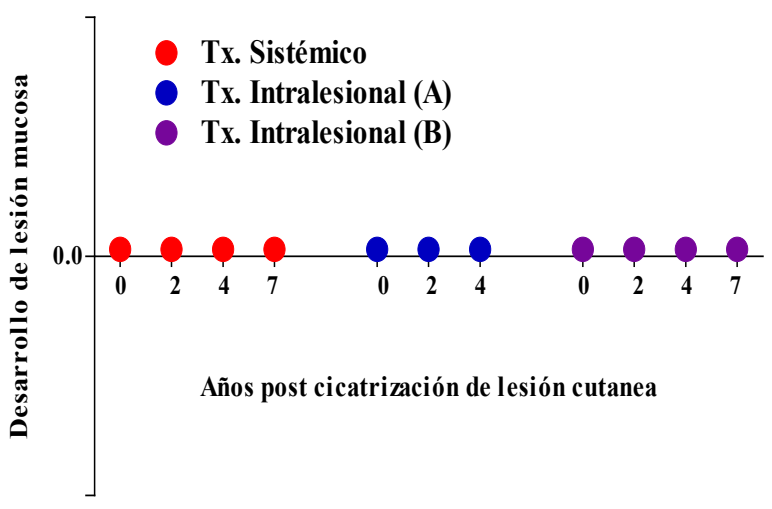

Respecto al seguimiento clínico postratamiento, tanto a aquellos que recibieron el tratamiento sistémico como el intralesional (3 y 6 aplicaciones respectivamente), la revisión clínica mostró la ausencia de lesiones mucosas posterior a la conclusión del tratamiento para Leishmaniasis cutánea independientemente del tratamiento realizado (Figura 2), así como también ausencia de otros compromisos cutáneos compatibles con falla terapéutica, presencia de recidivas y/o la aparición de nuevos episodios (Tabla 2).

Finalmente, respecto a los efectos adversos ocasionados por los tratamientos sistémicos o intralesional, el más frecuente fue el dolor en el lugar de la aplicación para ambos tratamientos empleados (sistémico o intralesional). Sin embargo, los casos que recibieron el tratamiento sistémico presentaron además mialgias, fiebre y cefalea en alrededor del $11 \%$ de los pacientes (Tabla 3). No se tienen datos de alteraciones hepáticas,

Tabla 2. Compromisos clínicos posteriores al tratamiento con antimoniales pentavalentes de pacientes con leishmaniasis cutánea. Los datos están expresados en frecuencia de casos. Los datos son expresados en frecuencia (\%)

\begin{tabular}{llccc}
\hline \multicolumn{1}{c}{ Variables } & & $\begin{array}{c}\text { Tx. } \\
\text { sistémico } \\
\end{array}$ & \multicolumn{2}{c}{ Tx intralesional } \\
Falla terapéutica & $\mathrm{Si}$ & $1(2.2)$ & $\mathrm{A}$ & $\mathrm{B}$ \\
& $\mathrm{No}$ & $45(97.8)$ & $11(100)$ & $9(100)$ \\
\hline Recidiva & $\mathrm{Si}$ & $1(2.2)$ & $1(9.1)$ & $\neg$ \\
& $\mathrm{No}$ & $45(97.8)$ & $10(90.9)$ & $9(100)$ \\
\hline Nueva Infección & $\mathrm{Si}$ & $1(2.2)$ & $\neg$ & $2(22.2)$ \\
& $\mathrm{No}$ & $45(97.8)$ & $11(100)$ & $7(77.8)$ \\
Nuevo Tratamiento para & $\mathrm{Si}$ & $2(4.4)$ & $\neg$ & $2(22.2)$ \\
leishmaniasis & $\mathrm{No}$ & $44(95.6)$ & $11(100)$ & $7(77.8)$ \\
\hline
\end{tabular}

Tx Sistémico $=$ Tratamiento convencional por 20 días de aplicación intramuscular dosis/kg/peso; Tx. intralesional (A) = Seis aplicaciones de medicamento intralesional; Tx intralesional $(\mathrm{B})=$ Tres aplicaciones de medicamento intralesional. 
Tabla 3. Efectos adversos presentados durante el tratamiento con antimoniales pentavalentes de pacientes con leishmaniasis cutánea. Los datos están expresados en frecuencia de casos. Los datos son expresados en frecuencia (\%)

\begin{tabular}{|c|c|c|c|c|}
\hline \multirow{2}{*}{\multicolumn{2}{|c|}{ Tipo de Efectos adversos }} & \multirow{2}{*}{$\begin{array}{c}\text { Tx. sistémico } \\
\mathrm{n}=46\end{array}$} & \multicolumn{2}{|c|}{ Tx. intralesional } \\
\hline & & & \multirow{2}{*}{$\begin{array}{c}\mathrm{A} \\
\mathrm{n}=11\end{array}$} & \multirow{2}{*}{$\begin{array}{c}\begin{array}{c}B \\
n=9\end{array} \\
9(100\end{array}$} \\
\hline \multirow{2}{*}{ Dolor Local } & $\mathrm{Si}$ & $45(97.8)$ & & \\
\hline & No & $1(2.2)$ & $2(18.2)$ & $\neg$ \\
\hline \multirow{2}{*}{ Mialgia } & $\mathrm{Si}$ & $5(10.9)$ & $\neg$ & $\neg$ \\
\hline & No & $41(89.1)$ & $11(100)$ & $9(100$ \\
\hline \multirow{2}{*}{ Fiebre } & $\mathrm{Si}$ & $5(10.9)$ & $\neg$ & $\neg$ \\
\hline & No & $41(89.1)$ & $11(100)$ & $9(100$ \\
\hline \multirow{2}{*}{ Cefalea } & $\mathrm{Si}$ & $6(13)$ & $\neg$ & $\neg$ \\
\hline & No & $40(87)$ & $11(100)$ & $9(100$ \\
\hline \multirow{2}{*}{ Anorexia } & $\mathrm{Si}$ & $1(2.2)$ & $\neg$ & $\neg$ \\
\hline & No & $45(97.8)$ & $11(100)$ & $9(100$ \\
\hline \multirow[t]{2}{*}{ Fatiga/Astenia } & $\mathrm{Si}$ & $2(4.4)$ & $\neg$ & $\neg$ \\
\hline & No & $44(95.6)$ & $11(100)$ & $9(100$ \\
\hline \multirow{2}{*}{ Nauseas } & $\mathrm{Si}$ & $1(2.2)$ & $\neg$ & $\neg$ \\
\hline & No & $45(97.8)$ & $11(100)$ & $9(100$ \\
\hline \multirow{2}{*}{ Urticaria } & $\mathrm{Si}$ & $2(4.4)$ & $\neg$ & $\neg$ \\
\hline & No & $44(95.6)$ & $11(100)$ & $9(100$ \\
\hline \multirow{2}{*}{ Sensación de ardor } & $\mathrm{Si}$ & $1(2.2)$ & $\neg$ & $\neg$ \\
\hline & No & $45(97.8)$ & $11(100)$ & $9(100$ \\
\hline \multirow{2}{*}{ Prurito } & $\mathrm{Si}$ & $1(2.2)$ & $\neg$ & $\neg$ \\
\hline & No & $45(97.8)$ & $11(100)$ & $9(100$ \\
\hline
\end{tabular}

Tx Sistémico = Tratamiento convencional por 20 días de aplicación intramuscular dosis $/ \mathrm{kg} /$ peso; Tx. intralesional $(\mathrm{A})=$ Seis aplicaciones de medicamento intralesional; Tx intralesional $(\mathrm{B})=$ Tres aplicaciones de medicamento intralesional.

cardiacas y pancreáticas por no tener disponibles las pruebas de laboratorio en los servicios médicos donde se realizaron los tratamientos.

\section{Discusión}

La evaluación clínica a largo plazo realizada a los pacientes tratados con antimoniales pentavalentes sistémicos e intralesionales evidencio la ausencia de complicaciones mucosas de la Leishmaniasis en todos los casos, independientemente del esquema de tratamiento administrado. Aunque son escasos los estudios en Latinoamérica que realicen el seguimiento a largo plazo de los pacientes tratados con antimoniales pentavalentes sistémicos o intralesionales, los pocos que hay mostraron similares resultados al de este estudio, destacando la ausencia de desarrollo de lesiones mucosas ${ }^{12-14}$. Aun cuando no están suficientemente esclarecidas las razones por las cuales se puede desarrollar complicaciones mucosas, diferentes evidencias apuntan a una desregulación del sistema inmune del individuo como un factor importante para este desarrollo ${ }^{15-18}$. En este sentido, el efecto inmunomodulador de los antimoniales pentavalentes es tan importante como su efecto leishmanicida. Los antimoniales pentavalentes modifican la respuesta inmune específica favoreciendo la activación de los macrófagos y por tanto, facilitando la fagocitosis de los parásitos intracelulares ${ }^{19}$. Por otra parte, en ausencia de un sistema inmune funcional, la respuesta a los antimoniales pentavalentes es reducida para la curación de las ulceras y la probabilidad de complicaciones mucosas aumenta $^{20}$, debido a la modificación de la respuesta inmune de tipo celular ${ }^{21,22}$ del individuo con Leishmaniasis.

Otro hallazgo importante, fue la constatación de ausencia de casos de recidivas o reinfecciones en el grupo de pacientes que recibió 3 aplicaciones intralesionales del medicamento y si bien se identificó la presencia de nuevas lesiones cutáneas en otras regiones anatómicas estas correspondían a un nuevo episodio.

Los efectos adversos producidos por los medicamentos, fueron más frecuentes entre los pacientes que recibieron tratamiento sistémico (Tabla 3), siendo los más habituales la mialgia, fiebre y cefalea, que probablemente responden a la toxicidad del antimonio o de los aditivos que contiene la formulación farmacéutica ${ }^{23,24}$. En cambio este tipo de efectos adversos en la aplicación intralesional son considerados $\operatorname{raros}^{25}$. El dolor local se presentó en todos los casos independientemente de si el tratamiento fue por aplicación sistémica o intralesional debido a la hipersensibilidad local del tejido inflamado por la toxicidad del antimonio ${ }^{26,27}$ y en caso de la aplicación intralesional además la inflamación responde a la actividad del parasito presente en el borde de las lesiones ${ }^{28,29}$.

Los resultados presentados en este estudio, tienen dos limitaciones relacionadas con tamaño de la muestra, debido a las dificultades de acceso a las comunidades, y el tiempo de seguimiento (4 a 7 años), que aun cuando es de largo plazo, no es el suficiente para descartar completamente el riesgo de que estos pacientes puedan desarrollar Leishmaniasis mucosa en un periodo mayor al evaluado como resultado de cambios en el comportamiento de su sistema inmunológico, ${ }^{15}$ que se pudieran desencadenar por múltiples causas. Sin embargo pese a las limitaciones señaladas, este es el primer estudio que se realiza con un seguimiento mayor de los cuatro años para este tipo de tratamiento en Latinoamérica.

\section{Conclusiones}

Este estudio es el primero en Bolivia que explora el efecto de los tratamientos intralesional y sistémico mediante un seguimiento clínico mayor a cuatro años, en el total de pacientes evaluados.

El tratamiento con antimoniales pentavalentes intralesionales es una opción confiable en relación a complicaciones futuras de la enfermedad, así como también en cuanto a su eficacia clínica ya que se alcanza un porcentaje similar de curación al obtenido por el tratamiento sistémico y respecto a su seguridad a largo plazo caracterizado por su escasa toxicidad, mínimo riesgo de recidivas, reinfecciones y por la ausencia de complicaciones mucosas refuerza las conclusiones que los consideran como una alternativa más costo-efectiva para los sistemas de salud en el manejo de la Leishmaniasis cutánea.

\section{Agradecimientos}

El estudio contó con el apoyo financiero de la fundación Damián. Los autores expresan su agradecimiento al personal de los centros de salud del parque Isiboro Sécure. Expresan igualmente sus agradecimientos al Dr. Miguel Guzmán-Rivero 
por su detallada revisión del manuscrito y sus sugerencias que contribuyeron a mejorar la redacción del mismo.
Conflicto de intereses: los autores declaran que no existe conflicto de intereses.

\section{Referencias bibliográficas}

1. Marzochi MCA,Marzochi KBF. Tegumentary and Visceral Leishmaniases in Brazil - Emerging Anthropozoonosis and Possibilities for Their Control. Cad. Saúde Públ. 1994 Julio; 10(2): 35975 .

2. Organización Panamericana de la Salud / Organización Mundial de la Salud. Manual de diagnostico y tratamiento de las Leishmaniasis. Asunción; 2018: 92.

3. Montalvo CM, Fraga J, Lianet Monzote C, García M, Fonseca L. Diagnóstico de la leishmaniasis: de la observación microscópica del parásito a la detección del ADN Leishmaniasis diagnosis: going from microscopic observation of parasite to DNA detection [Internet]. Vol. 64, Revista Cubana de Medicina Tropical. 2012

4. Soto J, Rojas-Cabrera E, Verduguez-Orellana A, Nena W, Maldonado M, et al. Intralesional Antimony for Single Lesions of Bolivian Cutaneous Leishmaniasis: Table 1. Clin Infect Dis. 2013 May 1;56(9):1255-60.

5. Rojas-Cabrera E, Verduguez-Orellana A, Cordova-Rojas M, Guzmán-Rivero M. Guia Operativa para el manejo de Leishmaniasis en Bolivia. Guía operativa. Cochabamba: CUMETROP, Cochabamba; 2017. Report No.: 1:2-6.

6. Organización Panamericana de la Salud. Manual de procedimientos para vigilancia y control de las leishmaniasis en las Américas. Washington, D.C.: OPS; 2019: 44-6

7. Tuon FF, Amato VS, Graf ME, Siqueira AM, Nicodemo AC, Neto VA. Treatment of New World cutaneous leishmaniasis - A systematic review with a meta-analysis. Vol. 47, International Journal of Dermatology. 2008:109-24.

8. Rodrigues AM, Hueb M, Santos TARR, Fontes CJF. Fatores associados ao insucesso do tratamento da leishmaniose cutânea com antimoniato de meglumina. Rev Soc Bras Med Trop. 2006 Apr;39(2):139-45.

9. Rojas-Cabrera E, Verduguez-Orellana A, Cordova-Rojas M, Guzmán-Rivero M. Antimoniato de meglumine perilesional en leishmaniasis cutánea con falla terapéutica sistémica: serie de casos. Gac Med Bol. 2019 Enero-Junio; 42(1): 74-8.

10. Ministério da Saúde Secretaria de Vigilância em Saúde. Departamento de Vigilância das Doenças Transmissíveis. Manual de vigilância da leishmaniose tegumentar. Brasilia; 2017. 74-6 p.

11. Blum J, Lockwood DNJ, Visser L, Harms G, Bailey MS, Caumes E, et al. Local or systemic treatment for New World cutaneous leishmaniasis? Re-evaluating the evidence for the risk of mucosal leishmaniasis. Int Health . 2012 Sep 1 ; 4(3):153-63.

12. Amato VS, Tuon FF, Imamura R, Abegão De Camargo R, Duarte MI, Neto VA. Mucosal leishmaniasis: Description of case management approaches and analysis of risk factors for treatment failure in a cohort of 140 patients in Brazil. J Eur Acad Dermatology Venereol. 2009 Sep;23(9):1026-34.

13. Oliveira-Neto M, Schubach A, Mattos M, Goncalves da Costa S, Pirmez C. Intralesional therapy of American cutaneous leishmaniasis with pentavalent antimony in Rio de Janeiro, Brazil - an area of Leishmania (V.) braziliensis transmission. Int J Dermatol. 1997 Jun 1;36(6):463-8

14. Sáenz RE, Paz HM, de Rodriguez GC, de Vásquez AM, Mata RE, Johnson CM. Mucocutaneous leishmaniasis in Panama. Etiologic agent, epidemiologic and clinical aspects. Rev Med Panama. 1989 Jan 14(1):6-15.

15. Gupta G, Oghumu S, Satoskar AR. Mechanisms of Immune Evasion in Leishmaniasis. In: Advances in Applied Microbiology. Academic Press Inc.; 2013. p. 155-84.

16. Carvalho LP, Passos S, Schriefer A, Carvalho EM. Protective and pathologic immune responses in human tegumentary leishmaniasis. Vol. 3, Frontiers in Immunology. 2012: 5-9.

17. Conceição-Silva F, Leite-Silva J, Morgado FN. The binomial parasite-host immunity in the healing process and in reactivatio $\mathrm{n}$ of human Tegumentary leishmaniasis. Vol. 9, Frontiers in Microbiology. Frontiers Media S.A.; 2018: 2-8.

18. Martínez-Valencia AJ, Daza-Rivera CF, Rosales-Chilama M, Cossio A, Casadiego-Rincón EJ, Desai MM, et al. Clinical and parasitological factors in parasite persistence after treatment and clinical cure of cutaneous leishmaniasis. PLoS Negl Trop Dis. 2017 Jul 13;11(7):8-11.

19. Van Griensven J, Carrillo E, López-Vélez $\mathrm{R}$, Lynen L, Moreno J. Leishmaniasis in immunosuppressed individuals. Vol. 20, Clinical Microbiology and Infection. Blackwell Publishing Ltd; 2014. p. 286-99.
20. Saldanha ACR, Romero GAS, Guerra C, Merchan-Hamann E, Macedo V de O. Estudo comparativo entre estibogluconato de sódio BP $88^{\circ} \mathrm{e}$ antimoniato de meglumina no tratamento da leishmaniose cutânea II. Toxicidade bioquímica e cardíaca. Rev Soc Bras Med Trop [Internet]. 2000 Aug [cited 2019 Apr 16];33(4):383-8.

21. Haldar AK, Sen P, Roy S. Use of Antimony in the Treatment of Leishmaniasis: Current Status and Future Directions. Mol Biol Int. 2011;2011:123.

22. Basu JM, Mookerjee A, Sen P, Bhaumik S, Sen P, Banerjee S, et al. Sodium antimony gluconate induces generation of reactive oxygen species and nitric oxide via phosphoinositide 3-kinase and mitogen-activated protein kinase activation in Leishmania donovani-infected macrophages. Antimicrob Agents Chemother. 2006 May;50(5):1788-97.

23. Sundar S, Chakravarty J. Antimony toxicity. Vol. 7, International Journal of Environmental Research and Public Health. 2010: 4267-77.

24. Vally H, Misso NLA, Madan V. Clinical effects of sulphite additives. Clin Exp Allergy 2009 Nov 39(11):1643-51.

25. Oliveira LF, Schubach AO, Martins MM, Passos SL, Oliveira R V., Marzochi MC, et al. Systematic review of the adverse effects of cutaneous leishmaniasis treatment in the New World. Vol. 118, Acta Tropica. 2011: 87-96.

26. Haldar AK, Sen P, Roy S. Use of Antimony in the Treatment of Leishmaniasis: Current Status and Future Directions. Mol Biol Int. 2011;2011:1-23.

27. Mohammadzadeh M, Behnaz F, Golshan Z. Efficacy of glucantime for treatment of cutaneous leishmaniasis in Central Iran. J Infect Public Health . 2013 Apr; 6(2):120-4.

28. Ramalho DB, Silva RE da, Senna MCR de, Moreira HSA, Pedras MJ, Avelar DM de, et al. Meglumine antimoniate intralesional infiltration for localised cutaneous leishmaniasis: a single arm, open label, phase II clinical trial. Mem Inst Oswaldo Cruz. 2018 Jun 21;113(9):180-200.

29. Brito NC, Rabello A, Cota GF Efficacy of pentavalent antimoniate intralesional infiltration therapy for cutaneous leishmaniasis: A systematic review. Vol. 12, PLoS ONE. Public Library of Science; 2017. 